\title{
The dynamics and shapes of a viscous sheet spreading on a moving liquid bath
}

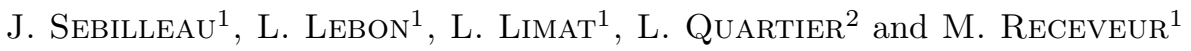 \\ 1 Laboratoire Matière et Systèmes Complexes, Université Paris Diderot, UMR705\%, 10 rue Alice Domon et Léonie \\ Duquet, 75205 Paris Cedex 13, France \\ 2 Laboratoire de Physique et de Mécanique des Milieux Hétérogènes (PMMH), UMR 857 of CNRS, ESPCI, Université \\ Pierre et Marie Curie and Université Paris Diderot, 10 rue Vauquelin, F-75231 Paris Cedex 05, France.
}

$\begin{array}{ll}\text { PACS } & 47.55 . \mathrm{nd} \text { - Spreading films } \\ \text { PACS } & 47.15 . \mathrm{gm} \text { - Thin film flows } \\ \text { PACS } & 47.55 . \mathrm{np} \text { - Contact lines } \\ \text { PACS } & 47.15 . \mathrm{km} \text { - Potentials flows }\end{array}$

\begin{abstract}
We investigate the shape and dynamics of a floating viscous sheet formed by a jet falling on a static or moving bath under partial wetting conditions. For a static bath, the viscous sheet has a circular shape and spreads with a uniform thickness that is surprisingly larger than the static Langmuir equilibrium thickness. This thickening effect seems to be linked to a peculiarity of the oil used for the bath, which is in situation of total wetting on the sheet surface, and climbs the sheet a bit like a macroscopic "precursor film" that increases dissipation at the sheet perimeter. For a moving bath, the viscous sheet evolves from an ellipse to a ribbon, a transient remarkable pear shape being observed between these two states. A simple kinematic model of advection of the spreading sheet by the bath predicts very well the characteristics of the ribbon regime. Convected sheets which shape is reminiscent of pendant drops in 2D are also observed at higher bath velocity, with interesting pinch off phenomena.
\end{abstract}

Introduction. - Contact line dynamics on solid substrates have been intensively studied these last decades [13]. There are comparatively much less works available on the equivalents on liquid susbtrates. Most of these studies are focussed on the spreading of liquid films in total wetting conditions $[4,5]$, a motivation being the application to environment problems and oil transport accidents. The case of partial wetting is even much less investigated though several works can be mentioned $[6,7]$ relative to the vibration modes of liquid/liquid contact lines or to high speed dewetting. This situation is unsatisfactory, as many applications (contact processes in chemical engineering [8], float techniques for glass production $[9,10]$, geophysics [11]) are concerned by questions such as those ones: what is the shape assumed by a floating sheet when interacting with more or les complex flows occuring in the bath, how does spreading or dewetting compete with advection by the bath, what are the stationary equilibrium shapes possible in the case of partial wetting, etc... In this paper we investigate the simple case of a highly viscous liquid impacting at constant rate a much less viscous bath be- fore to spread on its surface in partial wetting conditions, the bath being in turn flowing in a prescribed direction at a uniform and constant velocity. We explore the shape and thickness distribution assumed by the sheet, and show that there is, at long time scale, a possible stationary equilibrium shape (ribbon structure), which geometry can be deduced from a very simple model combining the flows associated to a point source and a uniform advection. This shape selection problem is reminiscent of the dewetting of a film flowing down a inclined solid surface : the flow along the incline breaks the natural axial symmetry of dewetting $[12,13]$ just as the flow in the moving bath breaks here the axial symmetry of spreading. This question is not only a fundamental challenge but also a problem of great technical interest. This situation is close to that encountered in the float process of glass fabrication, in which the liquid glass is poured at constant rate on a tin bath, itself flowing because of viscous shear stresses exerted by the glass plate extraction at the furnace exit [9].

In what follows, we first describe our experiment and the general sheet structure that we observed varying flow rate 
and bath velocity. We then investigate more quantitatively the spreading of the sheet on a static bath, showing that the sheet thickness selection is not a trivial problem. Contrary to intuition, a uniform thickness emerges that is not necessary that observed for a static floating sheet with zero expansion. In a third step, we investigate in more details the asymptotic ribbon structure observed at long time scales and precise its geometric properties in light of a very simple advection/spreading model of pure kinematic nature.

Experiments and general observations. - In our experiment (see fig. 1-(a)), the spreading liquid 1 (glycerin, viscosity ${ }^{1}$, mass density $\rho_{1}=1.261$ g.cm ${ }^{3}$ ) falls as a vertical jet and hits at a constant rate $Q$ (typically between $\mathrm{Q}=0$ and $25 \mathrm{~cm}^{3} \cdot \mathrm{s}^{-1}$ ), a bath of liquid 2 (perfluorinated silicon oil, Galden HT 270, viscosity $\eta_{2}=2.610^{-2}$ Pa.s, mass density $\rho_{2}=1.85$ g.cm ${ }^{3}$ ). The bath motion is imposed by an immersed rotating belt, which induces a surface velocity $\mathrm{U}_{b}$ ranging between 0 and $10 \mathrm{~cm} . \mathrm{s}^{-1}$. The three involved surface tensions $\gamma_{1}=59.5 \mathrm{mN}^{-1} \mathrm{~m}^{-1}, \gamma_{2}=25$ $\mathrm{mN} . \mathrm{m}^{-1}$ and $\gamma_{12}=26 \mathrm{mN} . \mathrm{m}^{-1}$ have been measured with the pendent drop method, and correspond to partial wetting conditions. The spreading parameter $\mathrm{S}=\gamma_{2}-\gamma_{1}-\gamma_{12}$ $=-60.5 \mathrm{mN} \cdot \mathrm{m}^{-1}$ is negative and, in static conditions, a finite volume of liquid 1 adopts on liquid 2 the structure of a floating sheet whose equilibrium thickness $h_{E} \simeq 5,6$ $\mathrm{mm}$ is given by Langmuir law:

$$
\frac{1}{2} \rho_{1} g \Delta h_{E}^{2}=\gamma_{1}+\gamma_{12}-\gamma_{2}
$$

where $\Delta=\left(\rho_{2}-\rho_{1}\right) / \rho_{2}$. This theoretical value is in good agreement with direct measurements that we performed on static floating sheets of constant volume ${ }^{2}$. In the dynamic case, we have investigated the shapes assumed by the liquid sheet, varying the flow rate and bath velocity. A typical set of pictures taken from above is reproduced on fig. 1-d to 1-h.

When the bath is at rest (picture $\mathrm{d}$ ), the impacting liquid expands as a circular sheet and its radius $R$ increases with time following a law of the kind:

$$
R(t)=\sqrt{\frac{Q t}{\pi h_{D}}}
$$

where $h_{D}$ is a dynamical mean thickness that we found appreciably larger than $h_{E}$ (typically $20 \%$ of increase). The lateral view, reproduced on picture (b) indicates that the thickness distribution is uniform except close to the injection and in the vicinity of the contact line, which implies that this mean thickness is in fact also the local value of the sheet thickness itself. The volume of liquid collected

\footnotetext{
${ }^{1}$ The nominal viscosity of glycerin at $20^{\circ} \mathrm{C}$ is $1 \mathrm{~Pa} . \mathrm{s}$ but, as glycerin is highly hygroscopic, its viscosity decrease rapidly to a value of $0.3 \mathrm{~Pa} . \mathrm{s}$ (measured with a cone/plate rheometer) $\eta_{1}=0.3 \mathrm{~Pa} . \mathrm{s}$

${ }^{2}$ we checked (1) on time scales small enough to neglect (typically less than $60 \mathrm{~s}$ ) the effect of the film issued from the bath, and that climbs the sheet, mentioned in the next section
}

in the "blob" under the impact is always less than $1 \mathrm{~cm}^{3}$ which represents less than $2 \%$ of the total injected volume. So this can not explain the observed difference between the dynamical mean thickness $h_{D}$ and the Langmuir equilibrium thickness $h_{E}$.

When the bath is put into motion the liquid sheets preserves its uniformity (fig. 1-(c)). At low velocity, the liquid sheet drifts with the bath while its centre stops to coincide with the impact point leading to an elliptical shape (fig. 1-(e)). At higher bath velocity, a transient pear shape is observed (fig. 1-(f)) that evolves at long time scales to a stationary ribbon shape (fig. $1-(\mathrm{g})$ ). The width $W$ of this ribbon and the position of the upstream front $L$ (see the fig. 1-(g)) saturate at constant values for long time scales. At even larger bath velocity, more complex behaviours are observed, in which a drifting sheet detaches periodically from a narrow floating ligament (fig 1-(h)) via a pinching instability very similar to that involved for gravity driven vertical jets [14], though here gravity is replaced by the shear exerted by the bath. We suggest here that the analogy could even be developed more formally for the sheet shape itself, that could result from a balance between an effective surface energy associated to the drop perimeter (at constant volume) and the effective gravity provided by the viscous shear exerted by the bath.

Spreading on a static bath. - Though reminiscent of other viscous sheet problems [10], the uniformity of the floating sheet thickness is not so trivial, as strong elongational viscous stresses should develop tangentially to the sheet. Also, the value of the thickness selected remains to be understood. We have thus investigated more carefuly the spreading on a static bath, recording radius of the sheet as a function of time for different flow rates. A typical plot is presented on fig. 2-a, on which the law $\mathrm{R}(\mathrm{t})$ of eq.(2) is plotted and compared to its equivalent for a thickness equal to Langmuir value (dark line). As one can see both laws behave as the square root of time, but the real one is always smaller than its Langmuir equivalent, which attests that $h_{D}$ is indeed always larger than $h_{E}$. We have also investigated what happens after suspending the injection at different times. As one can see on the same figure, the radius of the sheet slowly relaxes to its Langmuir equivalent, i.e. to the value that would have been deduced from the continuous line. Repeated experiments convinced us that the dynamic thickness $h_{D}$ is in fact independent of the flow rate $\mathrm{Q}$, at least for moderate time scales (typically $40 \mathrm{~s}$ ), the value found for glycerin being of order $h_{D} \simeq 7 \mathrm{~mm}$, to be compared with $h_{E} \simeq$ $5.6 \mathrm{~mm}$. This uniformity of the thickness distribution can be recovered theoretically by a model in which gravity expands the floating sheet against the elongational viscous stresses [15]. As the spreading liquid is highly viscous, the flow is assumed to be extensional and axisymmetric and is therefore fully described by the radial velocity $u=u(r, t)$ and the sheet thickness $h=h(r, t)$. For a radial extensional flow, the radial and azimuthal stresses are given 


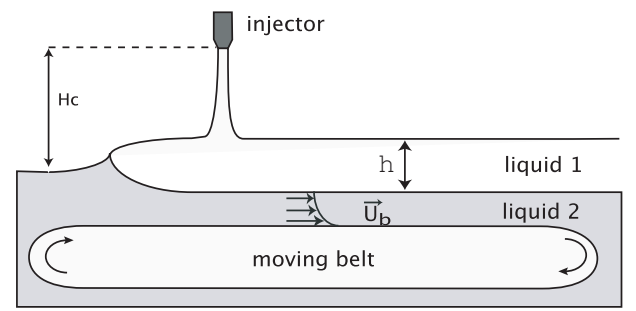

(a)
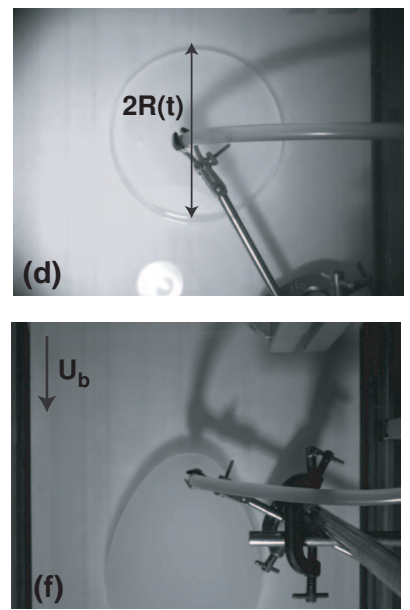

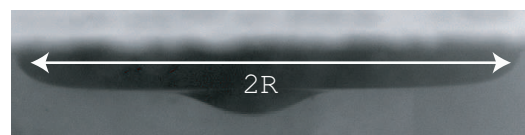

(b)

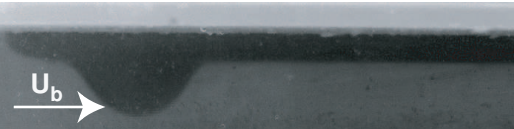

(c)
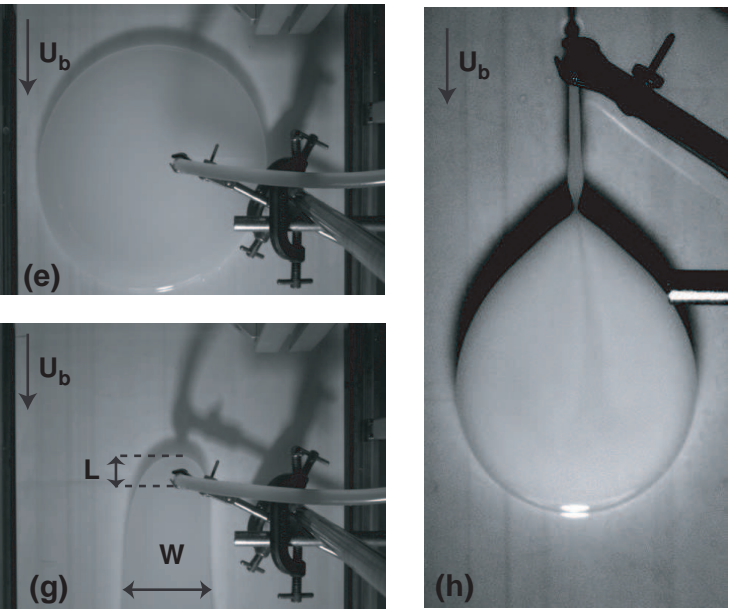

Fig. 1: (a) Principle of our experiment. A highly viscous liquid (glycerin) is poured from a vertical tube onto a free surface of a much less viscous liquid (perfluorinated silicon oil), itself flowing at constant speed under the action of an immersed rotating belt. The height of fall is $H_{c}=2 \mathrm{~cm}$ and the diameter of the injector is $\Phi=1 \mathrm{~cm}$. (b and c) Viewed from the side the floating sheet thickness is nearly uniform for both a static (b) or moving (c) bath, except in the close vicinity of the jet and of the contact line. ( $\mathrm{d}$ to g) Different shapes observed for the floating sheet: (d) Static bath $\mathrm{Q}=6.44 \mathrm{~cm}^{3} \cdot \mathrm{s}^{-1}$, (e) bath of low velocity $\mathrm{Q}=5.6$ $\mathrm{cm}^{3} \cdot \mathrm{s}^{-1}$ and $\mathrm{U}_{b}=0.2 \mathrm{~cm} . \mathrm{s}^{-1}$, (f) larger velocity at short time $\mathrm{Q}=8.5 \mathrm{~cm} 3 . \mathrm{s}^{-1}, \mathrm{U}_{b}=1 \mathrm{~cm} \cdot \mathrm{s}^{-1}$ and $\mathrm{t}=13 \mathrm{~s}$, (g) larger velocity at long time scale $\mathrm{Q}=8.5 \mathrm{~cm}^{3} \cdot \mathrm{s}^{-1} \mathrm{U}_{b}=1 \mathrm{~cm} \cdot \mathrm{s}^{-1} \mathrm{t}=35 \mathrm{~s}$. (h) In several cases, floating sheets whose shape is reminiscent of a pendant drop detach from a floating ligament by a remarkable pinching instability $\left(\mathrm{Q}=0.57 \mathrm{~cm}^{3} \cdot \mathrm{s}^{-1}, \mathrm{U}_{b}=0.32 \mathrm{~cm} \cdot \mathrm{s}^{-1}\right)$.
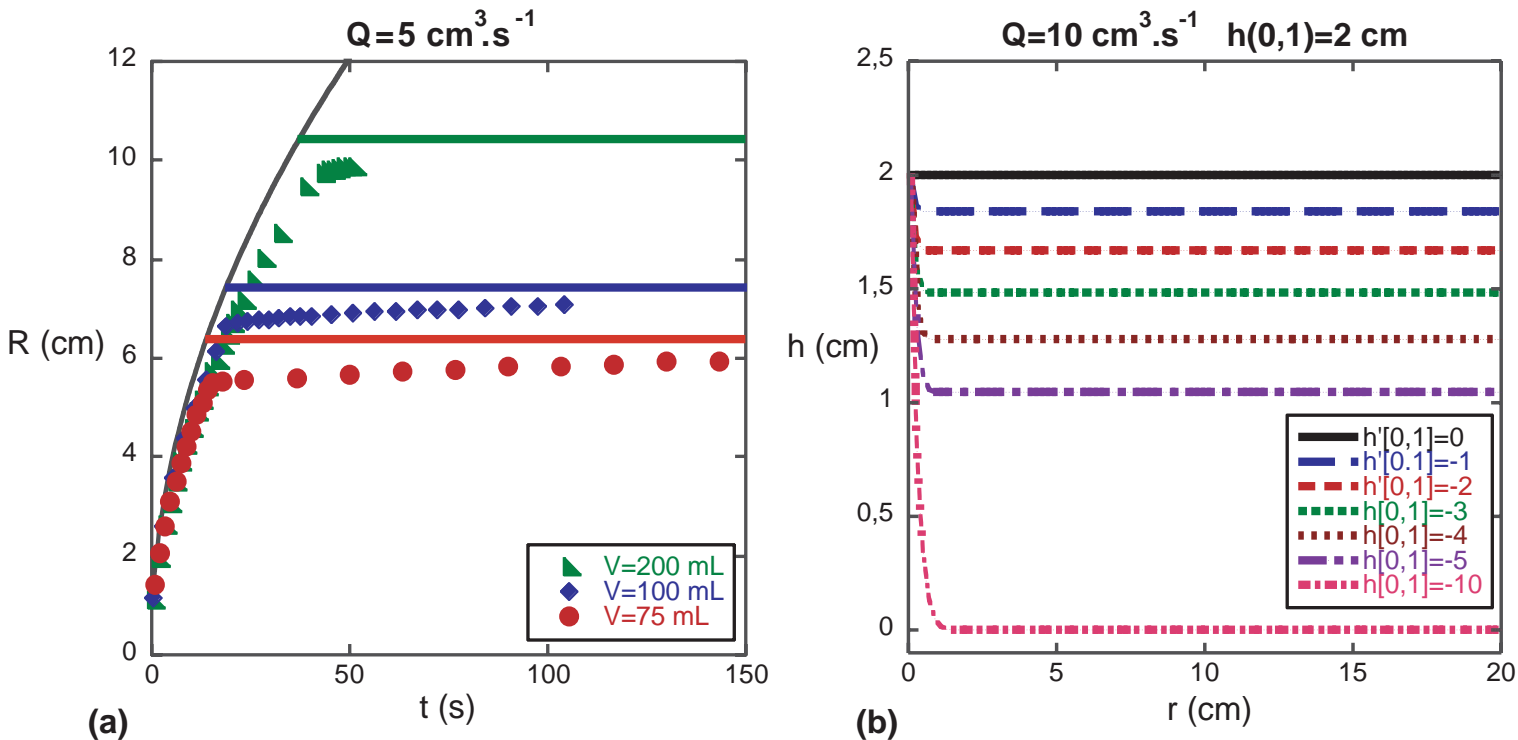

Fig. 2: (a) Typical experimental evolution of the radius of the spreading sheet $R$ with time for three different volumes $V$ injected during different times but with the same flow rate $Q=5 \mathrm{~cm}^{3} \cdot \mathrm{s}^{-1}$. It clearly appears that the spreading law differs for a spreading with a uniform thickness equal to the Langmuir thickness $h_{E}$ (dark line) though the radius continues to expand after the flow rate is stopped, in order that the final thickness ultimately reaches $h_{E}$. (b) Numerical integrations of eq. (8) for various "initial" conditions at $\mathrm{r}=0$, allowing us to recover the observed uniformity of the local thickness. 
respectively by :

$$
\sigma_{r r}=-p+2 \eta_{1} \frac{\partial u}{\partial r} \text { and } \sigma_{\theta \theta}=-p+2 \eta_{1} \frac{u}{r}
$$

and the continuity equation yields :

$$
\frac{1}{r} \frac{\partial[r u]}{\partial r}+\frac{\partial v_{z}}{\partial z}=0
$$

Assuming hydrostatic equilibrium and using the vanishing of the transverse stress $\sigma_{z z}$ at the free surface leads to :

$$
p=2 \eta_{1} \frac{\partial v_{z}}{\partial z}-\rho_{1} g(\Delta h-z)
$$

Combining this equation with the continuity equation (4) leads to :

$$
p=-2 \eta_{1} \frac{\partial[r u]}{\partial r}-\rho_{1} g(\Delta h-z)
$$

Ignoring surface tension and assuming a "plug" flow in the sheet, the balance of stress averaged over the thickness $h$ on a small element of length $d r$ and of angular opening $d \theta$ leads to :

$$
\frac{\partial}{\partial r}\left[r \int_{\Delta h-h}^{\Delta h} \sigma_{r r} d z\right]-\int_{\Delta h-h}^{\Delta h} \sigma_{\theta \theta} d z=0
$$

In the stationary case, the mass conservation leads directly to $u=Q /(2 \pi r h)$ and $(7)$ becomes :

$$
\frac{2}{h(r)}\left(\frac{\partial h(r)}{\partial r}\right)^{2}+\frac{1}{r} \frac{\partial h(r)}{\partial r}-2 \frac{\partial^{2} h(r)}{\partial^{2} r}=\frac{r h(r)^{2}}{L_{v}^{4}} \frac{\partial h(r)}{\partial r}
$$

where $\left.L_{v}=\left(2 Q \nu_{1} /(g \pi \Delta)\right)\right)^{1 / 4}$ is a millimetric length. A numerical analysis (with a Runge Kutta method of order 4), presented on fig 2-(b), shows that irrespectively of initial conditions at $\mathrm{r}=0$, all the solutions lead to a uniform thickness at large $r$. Its value $h_{D}$ is presumably ruled by the mechanical equilibrium condition at the external boundary $\mathrm{r}=\mathrm{R}\left(\mathrm{t}\right.$ ) (that would impose $h=h_{E}$ ), possibly perturbed by a viscous friction exerted by the bath. To capture this second effect, we tried to include in the model the shear exerted below the sheet by the bath across a thin boundary layer developing at the liquid/liquid interface [15], but reasonable values of this boundary layers lead to negligible corrections (less than one per thousand) on the sheet thickness. We finally found the plausible explanation of this height difference $\left(h_{D}-h_{E}\right)$ by a careful experimental investigation of what happens on the upper surface of the sheet. In our case (glycerin on modified silicon oil), the situation is complicated by the fact that the liquid of the bath is itself in situation of total wetting on the floating liquid, as the related spreading coefficient $\tilde{S}=$ $\gamma_{1}-\gamma_{2}-\gamma_{12}=8.5 \mathrm{mN} . \mathrm{m}^{-1}$ is positive. This is illustrated on fig. 3-(a): at the typical scales of the experiment (say $20 \mathrm{~s}$ ), a film of liquid 2 climbs liquid 1 . The evolution of the length $l$ (see fig. 3-(a)) of this oil film appears to evolve as the square root of time (see fig. 3-(b)) for different flow rate which means that the velocity of this film $V$ should be constant $\left(V \simeq 1.1 \mathrm{~mm} . \mathrm{s}^{-1}\right)$. This effect introduces an additional permanent shear stress of order $\sigma=\alpha \eta_{2} V / e$ where $e$ is the thickness of the oil film $(e=1,61 \mu \mathrm{m})$ and $\alpha$ a coefficient of order unity. The thickness of the oil film $e$ can be precisely measured by looking to the interference pattern (obtained with a $\mathrm{Na}$ spectral lamp) close to the liquid 2 contact line where the liquid film height jump from zero to an almost constant value $e$. We found $e=1.61 \mu \mathrm{m}$ which is compatible with the observation of interferences with white light (meaning $e<1,9 \mu \mathrm{m}$ ). Then the balance on the edge becomes :

$$
\frac{1}{2} \rho_{1} g \Delta h^{2}=2 \gamma_{12}+\frac{\alpha \eta_{2} V L}{e}
$$

Where $L$ is the typical radial length of the variation of the free surface near the contact line (typically of order of the capillary length). As the maximum capillary length of our system is $l_{c}=2,2 \mathrm{~mm}$ (for the glycerin) we choose $L \simeq 3 \mathrm{~mm}$. This new balance leads to a correction to the spreading thickness $h_{D}-h_{E} \simeq 0.8 \mathrm{~mm}$ which is of the same order of magnitude than what we observe experimentally. Of course, this effect is expected to disappear for most practical applications, when the wetting of the bath on the floating liquid is partial.

Study of the ribbon regime. - We have investigated in details the ribbon regime displayed on fig. 1-(g). Typical recording of the formation of the upstream front are reproduced on fig. 4-(a). As one can check, there is a final stationary state which geometry is well described by the two lengths $L$ and $W$ of fig. 1-(g). We have been able to calculate their dependence upon flow rate and bath velocity by identifying the flow in the sheet to that of a point source immersed in a uniform flow, that reads:

$$
\vec{u}=\frac{Q}{2 \pi h r} \vec{e}_{r}+U_{b} \vec{e}_{x}
$$

where $\vec{e}_{r}$ and $\vec{e}_{x}$ are respectively unit vectors oriented respectively in the radial and downstream direction, $\mathrm{r}$ is the distance to the liquid impact. Identifying the contact line to the separatrix of this flow yields immediately the relationships:

$$
W=\frac{Q}{h U_{b}} \text { and } L=\frac{Q}{2 \pi h U_{b}}
$$

The first one is checked on fig. 4-(b), and a good agreement is obtained. The second one is also well satisfied by our experimental observations, the observed ratio $W / L$ ranging between 5.8 and 6.7 , to be compared with $2 \pi$ (see fig. 4-(c)). To our knowledge, despite its simplicity and its technical importance, it is the first time that this flow structure is explained by a analytical model.

We also performed similar experiments with honey $\left(\eta_{1}=15.5\right.$ Pa.s, mass density $\rho_{1}=1.428$ g.cm ${ }^{3}, \gamma_{1}=64$ $\mathrm{mN} . \mathrm{m}^{-1}$ ) as viscous floating liquid. In this case the floating sheet still expands with a uniform thickness larger $h_{D}$ 


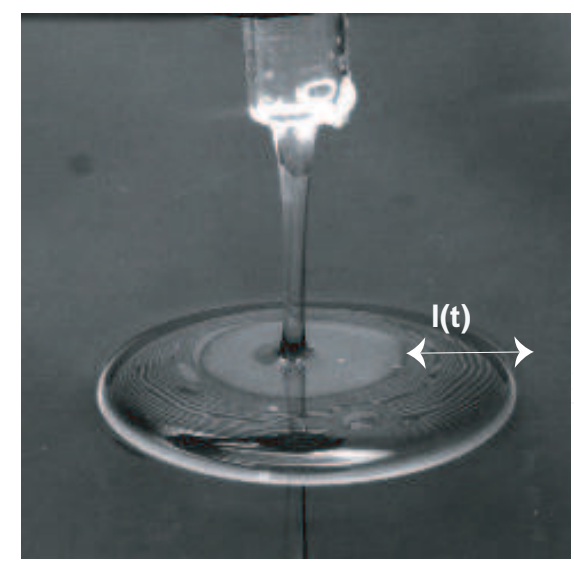

(a)

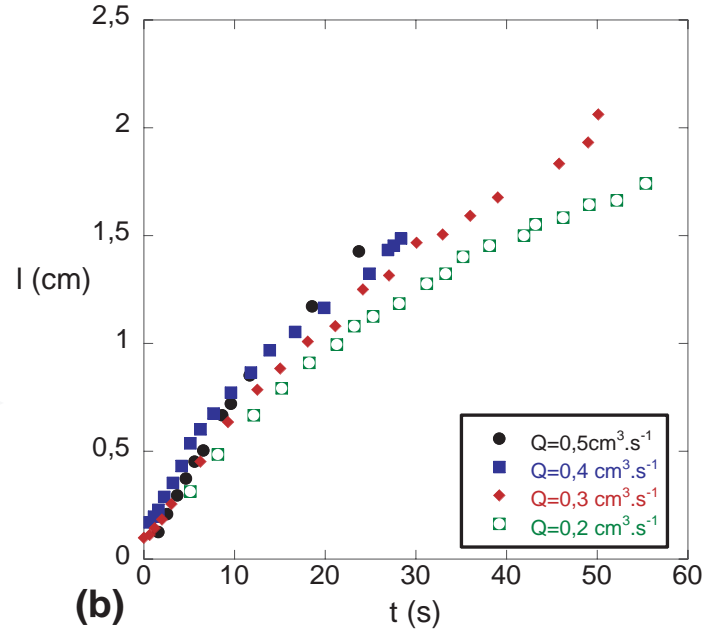

(b)

Fig. 3: (a) Picture of a static floating sheet: in our case (glycerol over perfluorinated silicon oil), the bath is able to climb the floating liquid as a macroscopic "precursor film". (b) Evolution of the length $l$ of the oil film climbing the floating liquid for different flow rate.
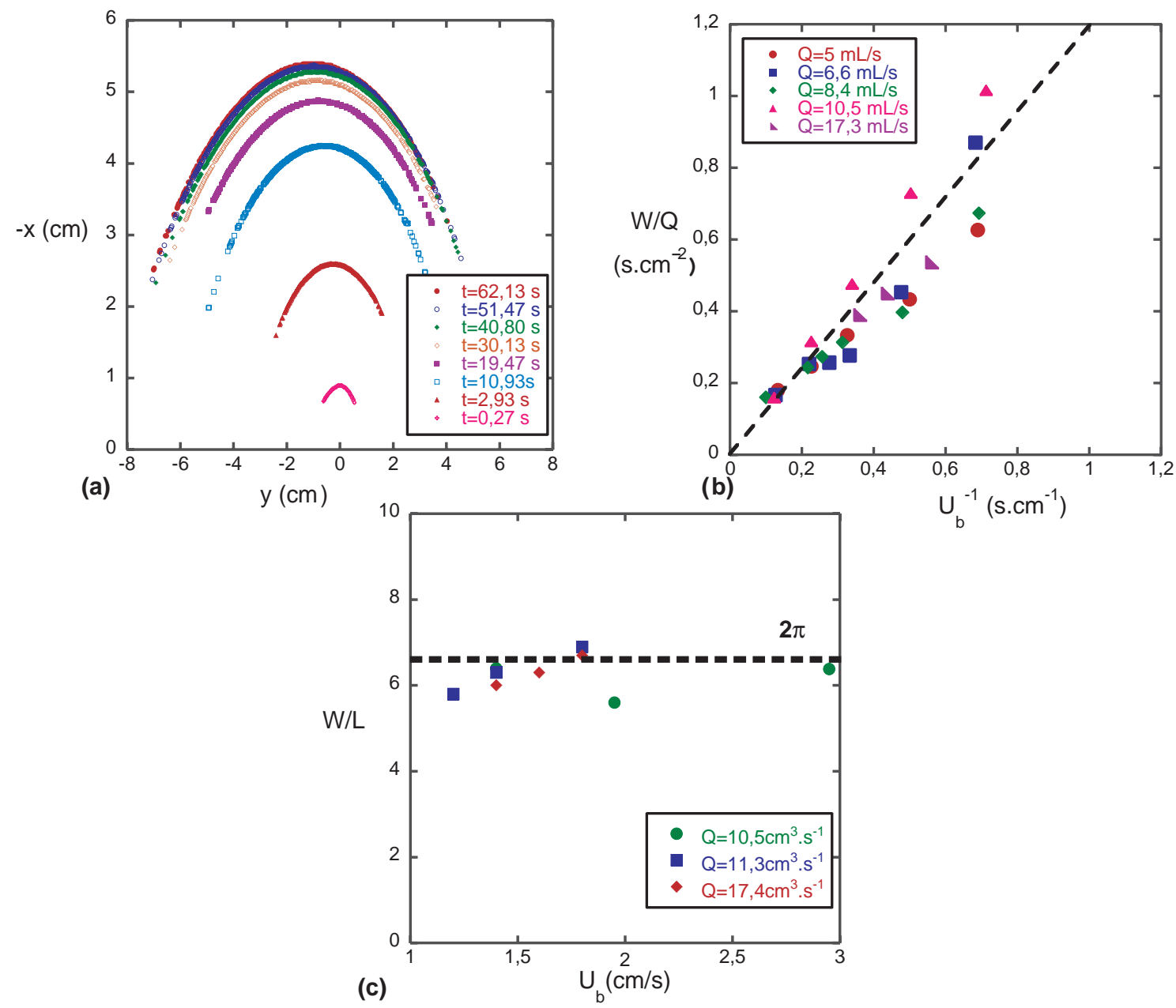

Fig. 4: (a): Evolution of the upstream front just after injection of the viscous fluid begins on the moving bath. There is clearly a asymptotical stationary shape. (b): Check of the law (11) linking the ribbon width to the flow rate Q and the bath velocity $U_{b}$. The value found for the slope of the dotted line is consistent with the thickness $h_{D}$ deduced from the growth on a static bath. (c) Several values of the ratio $W / L$ obtained by varying both the flow rate $\mathrm{Q}$ and the bath velocity $U_{b}$ 


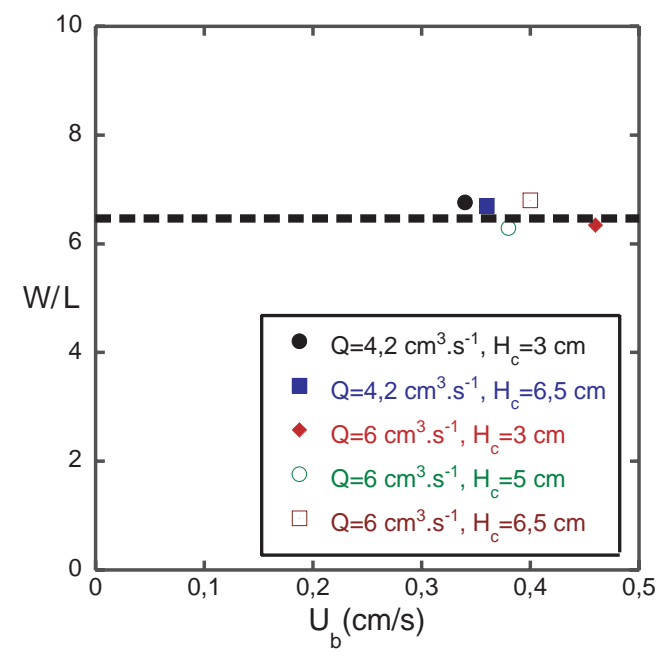

Fig. 5: Several values of the ratio $W / L$ obtained by varying both the flow rate $\mathrm{Q}$ and the height of fall $H_{c}$ and the velocity $U_{b}$ in the case of honey spreading on perfluorinated oil.

than the Langmuir equilibrium value, but this value appears to be influenced by both the wetting of oil and by the buckling of the honey when it impacts the oil bath (and so is dependent of the flow rate $Q$ and the height of fall $H_{c}$ ). This buckling also induced several spreading regimes which will be described in a further article. For these reasons it is quite difficult to check the evolution of the ribbon width with the bath velocity. But we can check the prediction for the ration $W / L$ which is independent of this spreading thickness $h_{E}$. As shown in fig.5, this ratio appears to be close to $2 \pi$ for different flow rate $Q$ and height of fall $H_{c}$ as predicted by our simple model.

Conclusion. - In this paper, we have investigated the shapes assumed by a viscous liquid expanding on a static or moving bath under partial wetting conditions. For a static bath, the spreading is radial with a uniform thickness $h_{D}$ larger than the static Langmuir equilibrium value. This over-thickness appears to be linked to a specific wetting property of the bath that climbs the spreading viscous sheet and, thus, changes the balance of forces on the sheet perimeter. For a moving bath, the viscous sheet shape evolves from an elliptic regime to a stationary ribbon regime separated by a transient pear shape regime. For the first time, the final ribbon regime is well characterized by a simple kinematic model of advection of the viscous sheet by the bath, which can be used to understand and ameliorate several technical applications. More generally our work opens new perspectives as more complex behaviors are also observed when one explores larger values of both flow rate and bath velocity, as for instance the "pendent sheet" regime of fig. 1-(h), that would deserve further investigations. Preliminary investigations on even more viscous fluids (not reported here) also revealed us other regimes mixing buckling of the impacting jet and spreading on the moving bath [15] that remain to be in- vestigated. In another direction, we believe that contact line dynamics on a moving bath should deserve more attention, especially when the contact line fluctuations are coupled with chemical reactions [8]. Finally the mechanism which selects a "macroscopic precursor film" on our floating sheets, instead of its microscopic equivalent on solids [2] remains an opened issue possibly connected to the more usual problem of spreading in total wetting conditions investigated long ago by Hoult and others.

$$
* * *
$$

We are indebted to B. Scheid who corrected a minor mistake in eq. ( 8), and to discussions with Y. Couder, J. Eggers, C. Goulas, S. Quiligotti.and H.-A. Stone.

\section{REFERENCES}

[1] P.-G. De Gennes, Rev. Mod. Phys., 57 (1985) 827-863

[2] L. Leger and J.-F. Joanny, Rep. Progr. Phys., 55 (1992) 431-486

[3] D. Bonn, J. Eggers, J. Indekeu, J. Meunier and E. Rolley, Rev. Mod. Phys., 81 (2009) 739-805

[4] D. P. Hoult, Annual Review of Fluid Mechanics, 4 (1972) 341-368

[5] J.A. FAY, Oil spreading on the sea, edited by D.P. Hoult 1969 , p. 53

[6] X. Noblin, A. Buguin and F. Brochard-Wyart, Langmuir, 18 (2002) 9350-9356

[7] X. Noblin, A. Buguin and F. Brochard-Wyart, Phys. Rev. Lett., 96 (2006) 156101.1-156101.4

[8] L. WeH, J. of Coll. Int. Sci., 227 (2000) 42-47

[9] L.A.B Pilkington, Proc. Roy. Soc. London, 314 (1969) $1-25$

[10] B. Scheid, S. Quiligotti, B. Tran, R. Gy and H. A. Stone, Eur. Phys. J. B, 68 (2009) 487-494

[11] C. Schoof, J. Fl. Mech., 573 (2007) 27-55

[12] T. Podgorski, J.-M. Flesselles, and L. Limat, Phys. Fluids, 11 (1999) 845-852

[13] J. Sebilleau, L. Lebon and L. Limat, Eur. Phys. J. Special Topics, 166 (2009) 139-142

[14] J. Eggers, Rev. Mod. Phys., 69 (1997) 865-930

[15] J. Sebilleau, PhD Thesis, University Paris 7 (2009) 